\title{
Structure and Optical Properties of Hybrid Metal-Dielectric Colloidal Photonic Crystals
}

\author{
A. V. Cvetkov' ${ }^{1}$, V. I. Gerbreders ${ }^{2}$, S. D. Khanin ${ }^{3}$, A. E. Lukin ${ }^{1}$, A. S. Ogurcovs ${ }^{2}$, \\ S. G. Romanov ${ }^{4}$, V. G. Solovyev', A. I. Vanin ${ }^{1}$, M. V.Yanikov ${ }^{1}$ \\ ${ }^{1}$ Department of Physics, Faculty of Physics and Mathematics, Pskov State University, Lenin Square 2, 180000 \\ Pskov, Russia; \\ ${ }^{2}$ G. Liberts' Innovative Microscopy Centre, Daugavpils University, Vienibas Street 13, LV-5400 \\ Daugavpils, Latvia; \\ ${ }^{3}$ Department of Physical Electronics, Faculty of Physics, Herzen State Pedagogical University of Russia, \\ Moyka-river embankment 48,191186 St. Petersburg, Russia; \\ ${ }^{4}$ Ioffe Physical Technical Institute, Polytechnicheskaya Street, 26, 194021 St. Petersburg, Russia
}

\begin{abstract}
Metal-dielectric nanocomposite optical materials based on colloidal crystals have been prepared by electrothermo-diffusion or magnetron sputtering of silver. Optical properties of these photonic crystals have been studied by angle-resolved reflectance and transmission spectroscopy. The interpretation of observed spectra has been made taking into account the Bragg diffraction, Fano resonance, Fabry-Perot resonance and surface plasmon-polaritons, which excitations contribute to the optical properties of plasmonic-photonic crystals.
\end{abstract}

Keywords: colloidal crystal, photonic crystals, photonic glasses, plasmonic crystal, nanostructures.

\section{INTRODUCTION}

Hybrid metal-dielectric photonic crystals have been fabricated on the basis of colloidal crystals [1] with the aim to design the next generation of optical materials.

Novel 3-dimensional $\mathrm{Ag} / \mathrm{opal}$ nanocomposites have been prepared by electro-thermo-diffusion of silver in opal templates [2]. Their reflectance spectra $\mathrm{R}_{\text {Ag/opal }}(\lambda)$ demonstrate the diffraction resonances. These resonances are red-shifted compared to those of $\mathrm{R}_{\text {opal }}(\lambda)$ spectra due to higher effective refractive index $n_{\text {Ag/opal }}>n_{\text {opal }}$. The most striking observation is the pronounced distortion of diffraction resonance band in reflectance spectra of Ag/opal nanocomposite with high metal content that contrasts to almost symmetric Bragg resonance shape of a bare opal. This phenomenon has been interpreted in [2, 3] as the manifestation of the Fano resonance $[4,5]$ between zero-order diffracted electromagnetic waves and those resonantly scattered by silver dendrites in Ag / opal composite.

Alternative approach to metal-dielectric architectures assumes integration of continuous metal films with colloidal crystals [6]. In particular, slab 2dimensional photonic crystals represented by monolayers (ML) of spheres have been sandwiched between flat and corrugated thin metal films that terminate their bottom and the top sides, respectively, to produce $A g / M L / A g$ architecture. Further this structure has been equipped with a corrugated FabryPerot microcavity, by adding a silica film and another metal film on top of $A g / M L / A g$ to achieve Ag/cavity/Ag/ML/Ag hybrid. The role of the metal layers here is two-fold - to form a microresonator across the structure and to guide surface plasmon polaritons (SPP) along the structure. This approach can be considered as the prototype fabrication technology for the optical chip production.

\section{MATERIALS AND METHODS}

Synthetic opals under study consist of silica $\left(\mathrm{SiO}_{2}\right)$ beads that are self-assembled in closely packed face centered cubic (FCC) lattice [7].

Silica opal samples were characterized by «VEGA // LMU Tescan» Scanning Electronic Microscope (SEM). According to SEM images, the mean sphere diameter of the studied opal is $D \approx 280 \mathrm{~nm}$. Moreover, some lattice disorder takes place (Fig. 1).

The electro-thermo-diffusion of silver in the $0.8 \mathrm{~mm}$ thick opal slab has been carried out under electric field of $\mathrm{E}=3.75 \mathrm{kV} / \mathrm{cm}$ at temperature $\mathrm{T} \approx 800 \mathrm{~K}$. 


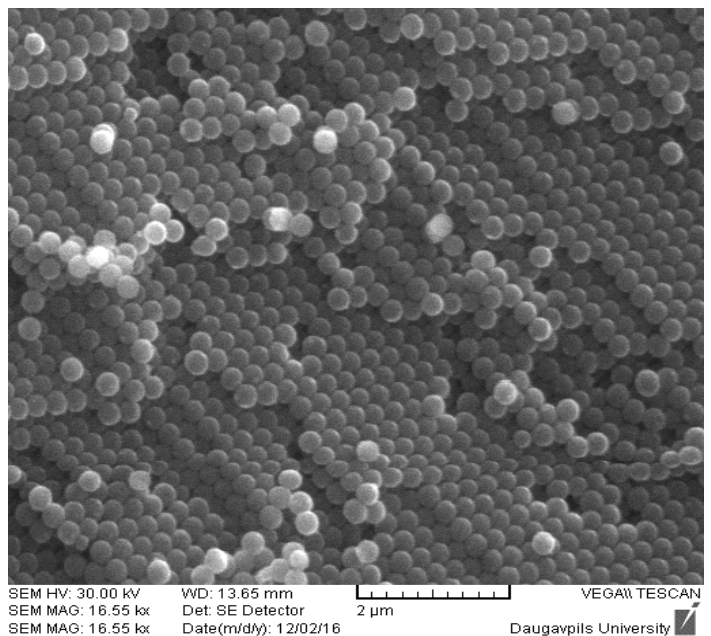

Fig. 1. SEM image of the silica opal matrix under study.

Hexagonally packed monolayer-based metaldielectric colloidal crystals $A g / M L / A g$ and $A g /$ cavity/Ag/ML/Ag have been prepared by magnetron sputtering of $A g$ in ATC ORION SERIES SPUTTERING SYSTEM [6] and characterized by "ZEISS FIB-SEM GEMINI" Scanning Electronic Microscope.

Angle-resolved reflectance and transmission spectra of the parent opal template and metaldielectric nanocomposites have been measured using white light illumination from a tungsten lamp. Spectra have been acquired by USB650 Red Tide spectrometer (Ocean Optics).

\section{RESULTS AND DISCUSSION}

Figure 2 shows SEM image of the silver infiltrate in one of the defect regions of the $A g /$ opal composite, confirming infiltration of opal. Silver concentration within these defect regions (e.g., near microscopic cracks, grain boundaries etc.) may be rather high (up to few atomic \%).

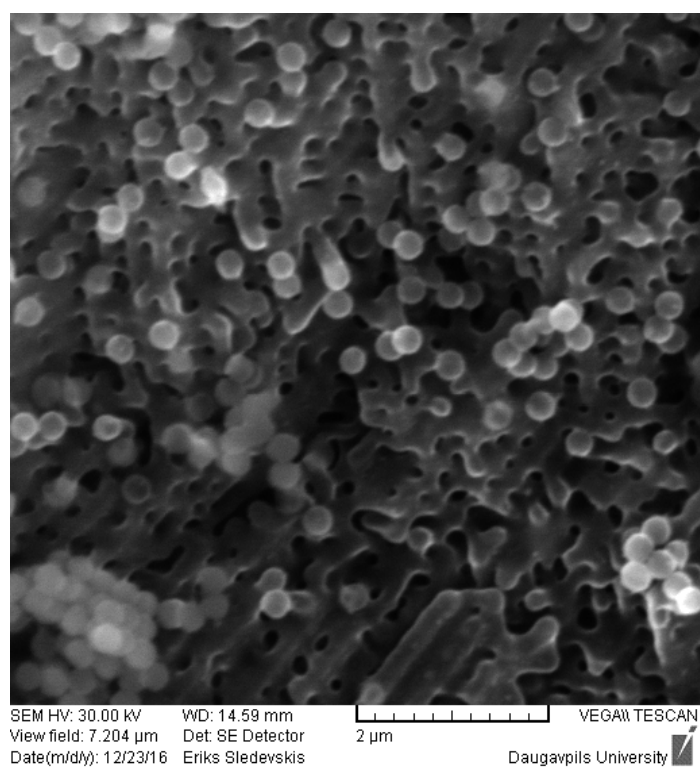

Fig. 2. SEM image of the defect region of the Ag/opal composite.
Reflectance spectra of opal template and those of nanocomposite Ag / opal at two different angles of light incidence are shown in Fig. 3.

One can see three maxima in the reflectance spectra of the opal template (Fig. 3, curves 1,2), but only one of them (the left one in Fig. 3) demonstrates distinct angular dispersion. At small angles, the resonance wavelength is $\lambda \approx 2 d n \approx 600 \mathrm{~nm}$, where $d=0.816 D$ is the interplane distance of (111) planes in FCC lattice and $n$ represents the effective refractive index of the photonic crystal. Hence, the maximum centred at $\sim 600 \mathrm{~nm}$ can be attributed to the zero-order (111) Bragg diffraction resonance.

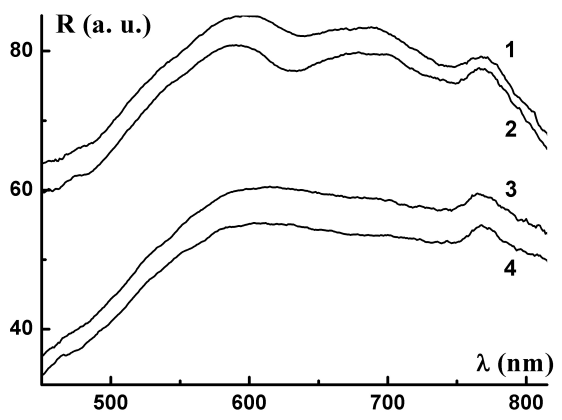

Fig. 3. Reflectance spectra of silica opal template $(1,2)$ and those of nanocomposite $\mathrm{Ag} / \mathrm{opal}(3,4)$ at the angles of incidence $25^{\circ}$ $(1,3)$ and $30^{\circ}(2,4)$.

The positions of two other maxima (near $690 \mathrm{~nm}$ and $770 \mathrm{~nm}$ - see Fig. 3) seem insensitive to the angle of light incidence. These maxima are presumably attributed to light scattering in partially disordered opal lattice structure ("photonic glass" [8] - [10]).

The diffraction band in the reflectance spectra of Ag / opal composite (Fig. 3, curves 3, 4) differs from the Bragg resonance shape in the spectra of a bare opal (Fig. 3, curves 1,2). As we already mentioned in Section I, this phenomenon is the manifestation of the Fano resonance [2] - [5] between two flows of electromagnetic waves: one is diffracted in the photonic crystal and the other is resonantly scattered by silver dendrites.

Figure 4 demonstrates SEM image of the hybrid colloidal plasmonic-photonic crystal [6]. This image shows the $A g / M L / A g$ hybrid integrated with $\mathrm{Ag} /$ cavity/ $\mathrm{Ag}$ resonator that is formed on top of the monolayer surface. 


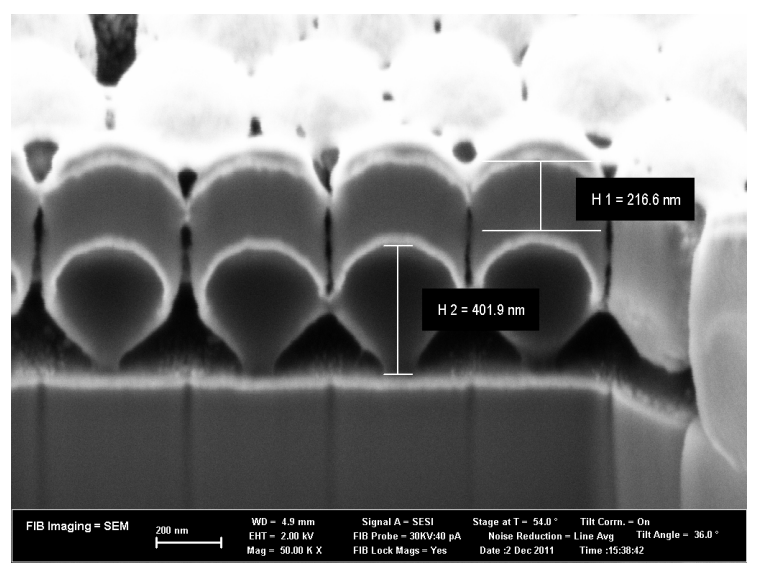

Fig. 4. SEM image of the focused ion beam cut of the $\mathrm{Ag} /$ cavity/Ag/ML/Ag hybrid colloidal plasmonic-photonic crystal under study [6].

The bare monolayer of spheres shows several resonances that correspond to the diffraction of guided resonances propagating along the monolayer (Fig. 5a, curve 1). Sandwiching a monolayer between $\mathrm{Ag}$ films results in the surprisingly weak transmission reduction, which is counteracted by two extraordinary transmission peaks (EOT) at 596 and $489 \mathrm{~nm}$. These peaks appear due to excitation of surface plasmon polaritons (SPPs) propagating at the interfaces of flat and corrugated silver films with ML and air, respectively (curve 2). The transmission enhancement manifests the enhancement of the optical density of states in surface plasmon polaritons. Peak at $400 \mathrm{~nm}$ is due to the intraband transitions of silver. Curve 5 in Fig. 5b illustrates relative changes introduced by metal films represented by the ratio $r_{5}(\lambda)=T_{2}(\lambda) / T_{1}(\lambda)$.

If another resonator $\mathrm{Ag} /$ cavity/ $\mathrm{Ag}$ with resonances at 770 and $385 \mathrm{~nm}$ (curve 3, Fig. 5a) is series connected to $A g / M L / A g$ one, the transmission is further reduced, but this reduction occurs selectively stronger at the Fabry-Perot resonances of the cavity (curve 4). Remarkably, the transmission peaks of the microcavity are converted in transmission minima of the hybrid architecture. This is clearly illustrated by the ratio $r_{6}(\lambda)=T_{4}(\lambda) /\left(T_{2}(\lambda) \times T_{3}(\lambda)\right)$, which demonstrates further enhancement of the EOT peaks and minima for cavity resonances (curve 6 in Fig. 5b). This effect can be tentatively interpreted as the extraordinary absorption (EOA). It occurs due to excitations of SPPs in the corrugated cavity in contrast to SPP absence in a microcavity with flat mirrors. Overall, one and the same architecture is capable of both enhancing and suppressing light transmission. Since both effects relate to guided SPP resonances, one can say that the transmission is enhanced owing to "bright" SPPs, whereas the absorption is enhanced owing to the cavity-related activation of normally "dark" SPPs.

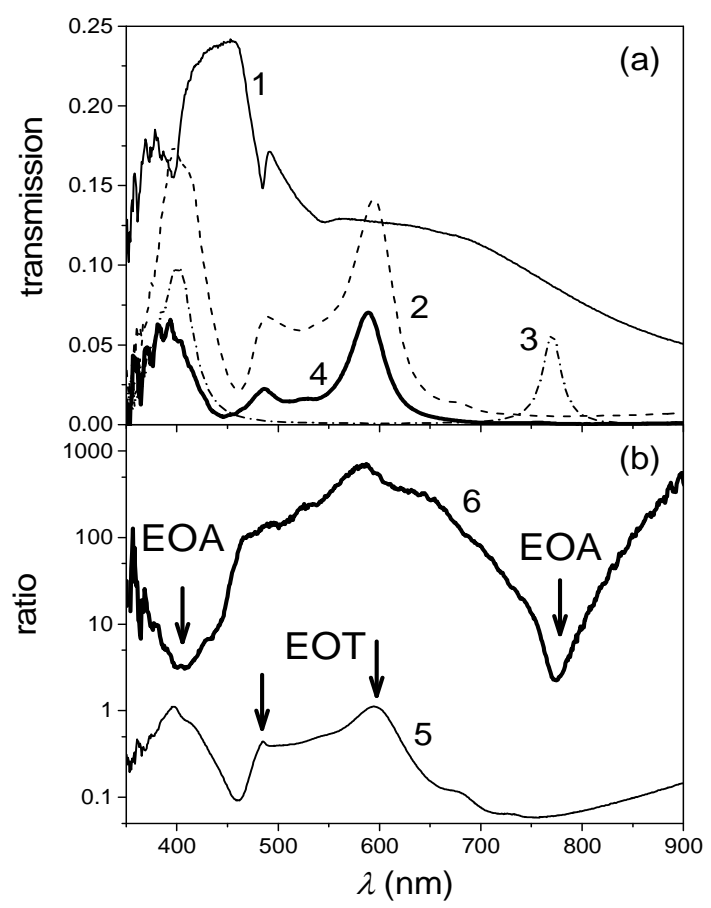

Fig. 5. (a) Transmission spectra of the monolayer $\left(T_{1}(\lambda)\right.$, curve 1), plasmonic-photonic crystal $A g / M L / A g\left(T_{2}(\lambda)\right.$ curve 2), microresonator $\left(T_{3}(\lambda)\right.$, curve 3$)$, plasmonic-photonic crystal with microresonator $A g /$ cavity / $A g / M L / A g,\left(T_{4}(\lambda)\right.$, curve 4) at the normal light incidence $\theta=0^{\circ}$. (b) The ratios $r_{5}(\lambda)=T_{2}(\lambda) / T_{1}(\lambda)$ (curve 5), $r_{6}(\lambda)=T_{4}(\lambda) /\left(T_{2}(\lambda) \times T_{3}(\lambda)\right.$ ) (curve 6).

\section{CONCLUSIONS}

The light diffraction in Ag / opal photonic crystals is affected by the light scattering at silver species. This interaction leads to Fano-type distortion of the Bragg resonance shape.

Engineering of optical properties of plasmonicphotonic crystals has been achieved via the architecture topology. Hybridization of different resonances results in either the extraordinary transmission or extraordinary absorption of light.

\section{ACKNOWLEDGEMENTS}

Authors thank M. I. Samoilovich for providing high-quality bulk opals, U. Peschel and D. Ploss for obtaining SEM images of a hybrid crystal.

This work was supported by the Ministry of Education and Science of Russian Federation under the "Development of Scientific Potential of Higher Educational Institutions" program and by German Academic Exchange Service (DAAD)

\section{REFERENCES}

[1] S. G. Romanov, A. V. Korovin, A. Regensburger, U. Peschel, "Hybrid colloidal plasmonic-photonic crystals", Advanced Materials, vol. 23, pp.2515-2533, 2011.

[2] V. L. Veisman, S. G. Romanov, V. G. Solovyev, M. V. Yanikov, "Optical properties of nanostructured silver embedded by electro-thermo-diffusion in opal photonic 
crystal", Environment. Technology. Resources: Proceedings of the 10th International Scientific and Practical Conference, Rezekne, Latvia, vol. 1, pp. 230-231, 2015.

[3] A. I. Vanin, V. G. Solovyev, "Modelling of Fano resonance in nanostructured material", Proceedings of VI International Conference on Photonics and Information Optics, Moscow, pp. 140-141, 2017.

[4] U. Fano, "Effects of configuration interaction on intensities and phase shifts", Phys. Rev., vol. 124, pp. 1866-1878, 1961.

[5] A. E. Miroshnichenko, S. Flach, Yu. S. Kivshar, "Fano resonances in nanoscale structures", Reviews of Modern Physics, vol. 82, pp. 2257-2298, 2010.

[6] S. G. Romanov, V. G. Solovyev, D. Ploss, U. Peschel, "Noninvasive management of photonic crystal resonances", Abstracts of the 4th International Topical Meeting on
Nanophotonics and Metamaterials, Seefeld, Austria, p. 26, 2013.

[7] V. G. Balakirev, V. N. Bogomolov, V. V. Zhuravlev Y. A. Kumzerov, V. P. Petranovskii ， S. G. Romanov, L. A. Samoilovich, "Three-dimensional superlattices in opals", Crystallography Reports, vol. 38, pp. 348-353, 1993.

[8] O. L. J. Pursiainen, J. J. Baumberg, H. Winkler, B. Viel, P. Spahn, T. Ruhl, "Nanoparticle-tuned structural color from polymer opals", Optics Express, vol. 15, pp.9553-9561, 2007.

[9] P. D. García, R. Sapienza, C. Lo'pez, "Photonic glasses: A step beyond white paint", Advanced Materials, vol. 22, pp. 12-19, 2010.

[10] Y. Takeoka, "Angle-independent structural coloured amorphous arrays", Journal of Materials Chemistry, vol. 22, pp. 23299-23309, 2012. 